\title{
Response effort and interresponse time: Effect of additional, response-produced stimulus feedback
}

\author{
HARVARD L. ARMUS and DENISE C. MIKESELL \\ The University of Toledo, Toledo, Ohio
}

\begin{abstract}
Previous studies have shown that greater response effort results in a larger percentage of short interresponse times in an operant task. An explanation, based on differential response-produced stimulus feedback resulting from different degrees of response effort, was proposed. An indirect test of this explanation showed that it was not adequate to account for the findings, and an alternate explanation, based on frustration, was proposed.
\end{abstract}

Previous operant studies with rats have shown that greater response effort (higher lever-operating-force requirements) results in a larger percentage of rapid response repetitions (Armus, 1986, 1988; Armus \& Mikesell, 1988). One explanation for these findings involves the notion of differential response-produced stimulus feedback. If stimulus feedback from a leverpress provides an important cue for the next leverpress, it is possible that more salient feedback cues are more strongly associated with leverpressing than less salient cues are. If so, and if feedback cues from a more effortful response are more salient than those from a less effortful response, such stronger association might result in a higher percentage of short interresponse times (less than $1 \mathrm{sec}$ ).

Although there is indirect support for this explanation, there are no data involving direct manipulation of response-produced feedback stimuli independent of response effort. In the present study, we attempted to do this by means of a compound, external, response-produced feedback stimulus. It was assumed that the provision of such a highly salient stimulus would reduce the differential effects of response-produced feedback stimulation from the different effort conditions.

\section{METHOD}

The subjects were 20 naive, male Long-Evans rats (Blue Spruce Farms), approximately 150 days old. The apparatus was an operant chamber approximately $30 \mathrm{~cm}$ on a side, fitted with a $10-\mathrm{cm}$-wide lever mounted parallel to the front wall of the chamber, $11 \mathrm{~cm}$ above the floor. Vertical movement of $4 \mathrm{~mm}$ was sufficient to operate the lever microswitch. The lever, which was heavily damped to prevent lever bounce, could be fitted with easily removable counterweights to change the response-effort requirement.

All animals were given 16 days of adaptation to a feeding schedule of $1 \mathrm{~h}$ of food every $24 \mathrm{~h}$ with ad lib access to water. The animals were

This research was partly supported by a grant from the Small Grants Program of the Graduate School of the University of Toledo, awarded to the senior author. Reprints may be requested from H. L. Armus, Department of Psychology, University of Toledo, 2801 West Bancroft Street, Toledo, OH 43606. then given 12 daily pretraining sessions of a maximum duration of $20 \mathrm{~min}$, during which leverpresses were reinforced on a continuous reinforcement schedule by one 45 -mg Noyes rat food pellet. The lever operating force was set at $10 \mathrm{~g}$. A total of approximately 575 reinforced presses was made by each rat during this pretraining phase.

The animals were then divided into two equal groups, matched on the basis of time to complete 100 responses on each of the last 2 pretraining days. For training, one of these groups was randomly assigned to the external stimulus-feedback condition (Group SF) and the other, to the no external stimulus-feedback condition (Group No SF). The additional stimulus feedback was provided by a Radio Shack buzzer (Archer 373-060A) mounted on the outside of the operant chamber lid, by two 7-W, 24-V clear lamps also mounted on the transparent lid, and by a white-jeweled 7-W, 24-V lamp mounted on the front wall of the chamber just above the lever. For Group SF, this compound stimulus remained on for the duration of each leverpress, thus providing additional external stimulus feedback of equal duration to the proprioceptive feedback resulting from a leverpress. Group No SF received no such external stimulus feedback.

The test phase was carried out over the next 12 days. On each test day, each rat was placed into the operant chamber for $16 \mathrm{~min}$, which were divided into two 8-min periods, each with a different responseeffort requirement (10 or $55 \mathrm{~g}$ ).

At the end of the first 8-min period, the houselight went off, and the experimenter immediately replaced the lever counterweight with the second one and reset the apparatus. This procedure took no longer than $3 \mathrm{sec}$. The order of response-effort requirements was the same for each rat on a given day but was varied over days, so that, over the 12 testing days, each force requirement appeared in each position an equal number of times and preceded and followed every other force requirement an equal number of times. Reinforcement was continued over the first 8 days of testing, while the last 4 days were run under extinction conditions.

\section{RESULTS AND DISCUSSION}

Analyses of variance were carried out on the data from the acquisition and extinction test phases. As can be seen in Figure 1, there seem to be small effects of both effort and external stimulation during the first half of acquisition, where the effort effect is typically stronger. However, neither of these main effects nor their interaction was significant over the entire acquisition phase or for the first half of acquisition.

In extinction, the effect of response effort was significant $[F(1,38)=9.24, p<.005]$. Although the effect of 


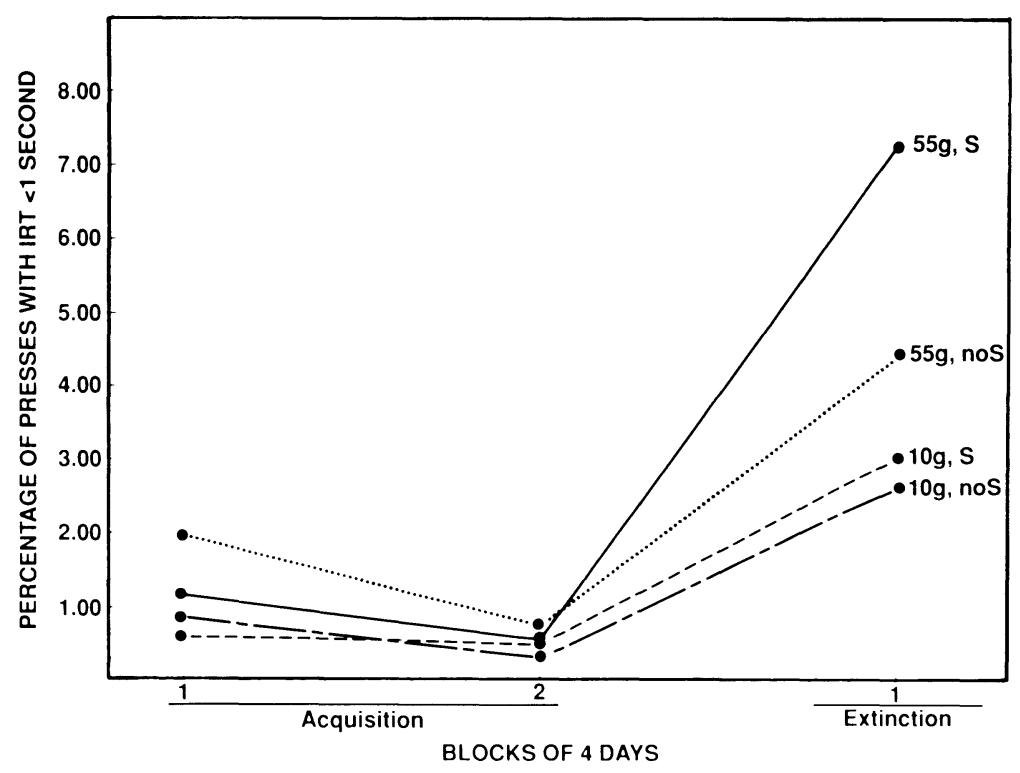

Figure 1. Percentage of presses with interresponse times of less than 1 sec over blocks of 4 test days in acquisition and extinction.

external stimulus feedback was also significant $[F(1,38)$ $=4.40, p<.05]$, the interaction of response effort and stimulus feedback was not.

Considering the extinction findings, it is obvious that there is a strong and predicted effect of response effort. However, it is equally obvious that this effect is not reduced by the provision of external, response-associated, stimulus feedback. The data, in fact, are in the direction opposite to this prediction.

These findings cast doubt on the previously proposed differential stimulus-feedback explanation for the effort effect, but they do suggest another possibility. It is possible that the response-effort effect is motivational in nature. The frustration resulting from a change from a less effortful to a more effortful response might bring about greater arousal (drive) and thereby facilitate rapid response repetition. This effort effect might very well be accentuated by the addition of extinction-produced frustration, resulting in a greater effect in extinction than in acquisition. And, finally, the addition of a possibly aversive external stimulus might interact with the arousal produced by the two sources of frustration to bring about an even stronger effect.

\section{REFERENCES}

ARMUS, H. L. (1986). Effect of response effort requirement on frequency of short interresponse times. Bulletin of the Psychonomic Society, 24, 284-285.

ARMUS, H. L. (1988). Effect of response effort requirement on relative frequency of short interresponse times: CRF and FR-5 reinforcement schedules. Bulletin of the Psychonomic Society, 26, 139-40.

Armus, H. L., \& Mikesell, D. C. (1988). Effect of response effort requirement on percentage of short interresponse times under delayed and immediate reinforcement. Psychological Reports, 63, 943-946. 